\title{
A PERIODICITY PROPERTY OF ITERATED MORPHISMS
}

\author{
JUHA HONKALA ${ }^{1}$
}

\begin{abstract}
Suppose $f: X^{*} \longrightarrow X^{*}$ is a morphism and $u, v \in X^{*}$. For every nonnegative integer $n$, let $z_{n}$ be the longest common prefix of $f^{n}(u)$ and $f^{n}(v)$, and let $u_{n}, v_{n} \in X^{*}$ be words such that $f^{n}(u)=z_{n} u_{n}$ and $f^{n}(v)=z_{n} v_{n}$. We prove that there is a positive integer $q$ such that for any positive integer $p$, the prefixes of $u_{n}$ (resp. $v_{n}$ ) of length $p$ form an ultimately periodic sequence having period $q$. Further, there is a value of $q$ which works for all words $u, v \in X^{*}$.
\end{abstract}

Mathematics Subject Classification. 68Q45, 68R15.

\section{INTRODUCTION}

In the theory of D0L systems many deep decidability results are proved by showing that certain D0L systems or pairs of D0L systems have some kind of regular behavior. In this paper we investigate a regularity property encountered when we compare the iteration of a morphism on two different words. More precisely, let $f: X^{*} \longrightarrow X^{*}$ be a morphism and let $u, v \in X^{*}$ be words. For every nonnegative integer $n$, let $z_{n}$ be the longest common prefix of the words $f^{n}(u)$ and $f^{n}(v)$, and let $u_{n}$ and $v_{n}$ be words such that $f^{n}(u)=z_{n} u_{n}$ and $f^{n}(v)=z_{n} v_{n}$. Hence, the words $u_{n}$ and $v_{n}$ give the words $f^{n}(u)$ and $f^{n}(v)$ from the first position where the latter words differ. In [4] it was shown that for any positive integer $p$ the prefixes of length $p$ of $u_{n}$ (resp. $v_{n}$ ) form an ultimately periodic sequence. This result plays a key role in the solution of the DF0L language equivalence problem in [4]. In this paper we study this periodicity result in more detail. In particular, we prove that there is a period which works simultaneously for prefixes of any length and does not depend on the words $u$ and $v$.

Keywords and phrases. Iterated morphism, periodicity.

${ }^{1}$ Department of Mathematics, University of Turku, 20014 Turku, Finland;

juha.honkala@utu.fi

(c) EDP Sciences 2007 
We assume that the reader is familiar with the basics concerning iterated morphisms (see [5-7]). Notions and notations that are not defined are taken from these references.

\section{Definitions And Results}

Suppose $X=\left\{x_{1}, \ldots, x_{m}\right\}$ is an alphabet with $m \geq 1$ letters. If $w \in X^{*}$, then $\#_{x_{i}}(w)$ is the number of occurrences of the letter $x_{i}$ in $w$. The Parikh mapping $\psi: X^{*} \longrightarrow \mathbf{N}^{m}$ is defined by

$$
\psi(w)=\left(\#_{x_{1}}(w), \ldots, \#_{x_{m}}(w)\right), \quad w \in X^{*} .
$$

The length of a word $w \in X^{*}$ is denoted by $|w|$. The length of the empty word $\varepsilon$ equals zero. The first letter of a nonempty word $w \in X^{*}$ is denoted by first $(w)$.

If $u, v \in X^{*}$ are words and there is a word $w \in X^{*}$ such that $u w=v$, we say that $u$ is a prefix of $v$. If $u \in X^{*}$ and $p$ is a positive integer, we denote by $\operatorname{pref}_{p}(u)$ the prefix of $u$ having length $p$. If $|u|<p$, it is understood that $\operatorname{pref}_{p}(u)=u$. This notation is extended in a natural way for pairs of words. Hence, if $(u, v) \in X^{*} \times X^{*}$ we denote

$$
\operatorname{pref}_{p}(u, v)=\left(\operatorname{pref}_{p}(u), \operatorname{pref}_{p}(v)\right) \text {. }
$$

Two words $u, v \in X^{*}$ are called comparable (with respect to the prefix order) if $u$ is a prefix of $v$ or vice versa. The longest common prefix of $u$ and $v$ is denoted by $u \wedge v$. Further, we denote

$$
u * v= \begin{cases}(\varepsilon, \varepsilon) & \text { if } u \text { and } v \text { are comparable } \\ \left((u \wedge v)^{-1} u,(u \wedge v)^{-1} v\right) & \text { otherwise. }\end{cases}
$$

Next, let $\left(a_{n}\right)_{n \geq 0}$ be a sequence and let $q$ be a positive integer. We say that $q$ is a period of $\left(a_{n}\right)_{n \geq 0}$ if there is an integer $n_{0}$ such that

$$
a_{n+q}=a_{n} \quad \text { whenever } n \geq n_{0} .
$$

A sequence is called ultimately periodic if it has a period.

The following theorem gives a basic result concerning periodicities in D0L sequences (see $[2,3])$.

Theorem 2.1. Suppose $X$ is an alphabet, $f: X^{*} \longrightarrow X^{*}$ is a morphism and $u \in X^{*}$. There is a positive integer $q$ such that for any positive integer $p, q$ is a period of the sequence

$$
\left(\operatorname{pref}_{p}\left(f^{n}(u)\right)\right)_{n \geq 0}
$$

In this paper we study the iteration of a given morphism on two different words. If $f: X^{*} \longrightarrow X^{*}$ is a morphism, $u, v \in X^{*}$ are words and $p$ is a positive integer, we consider the sequence $\left(\operatorname{pref}_{p}\left(f^{n}(u) * f^{n}(v)\right)\right)_{n \geq 0}$. It was shown in [4] that these sequences are ultimately periodic. (In [4] it is assumed that $\psi(u)=\psi(v)$.) In this paper we will study these sequences in more detail. In particular, we will prove the following result. 
Theorem 2.2. Suppose $X$ is an alphabet and $f: X^{*} \longrightarrow X^{*}$ is a morphism. There is a positive integer $q$ such that whenever $u, v \in X^{*}$ are words and $p$ is a positive integer, then $q$ is a period of the sequence

$$
\left(\operatorname{pref}_{p}\left(f^{n}(u) * f^{n}(v)\right)\right)_{n \geq 0} .
$$

The proof of Theorem 2.2 is given in the following section. The proof uses two lemmas from [4], but otherwise we do not assume familiarity with [4].

Let now $f: X^{*} \longrightarrow X^{*}$ be a morphism and let $u, v \in X^{*}$ be words. For every nonnegative integer $n$, define $z_{n}=f^{n}(u) \wedge f^{n}(v)$ and let $u_{n}, v_{n} \in X^{*}$ be words such that $f^{n}(u)=z_{n} u_{n}$ and $f^{n}(v)=z_{n} v_{n}$. Then there is a positive integer $q$ such that for any positive integer $p, q$ is a period of the sequences

$$
\left(\operatorname{pref}_{p}\left(u_{n}\right)\right)_{n \geq 0} \quad \text { and } \quad\left(\operatorname{pref}_{p}\left(v_{n}\right)\right)_{n \geq 0} \text {. }
$$

To deduce the existence of $q$ from Theorem 2.2, choose a new letter $\$$, extend $f$ by $f(\$)=\$$ and consider the words $u \$$ and $v \$$.

\section{Proofs}

We will prove Theorem 2.2 in two steps. The first subsection contains the main part of the proof. Without loss of generality we assume that $X$ contains at least two letters.

\subsection{Proof of Theorem 2.2 - a special CASE}

If $f: X^{*} \longrightarrow X^{*}$ is a morphism, a letter $a \in X$ is called bounded (with respect to $f$ ) if the length sequence $\left(\left|f^{n}(a)\right|\right)_{n \geq 0}$ is bounded above by a constant. If $a \in X$ is not bounded, then $a$ is called growing. A word $w \in X^{*}$ is called bounded if no letter of $w$ is growing.

In this subsection we assume that $f: X^{*} \longrightarrow X^{*}$ is a morphism which has the following additional properties:

(i) There is a positive integer $K$ such that if $x, y \in X^{*}$ are nonempty words and $\operatorname{first}(x) \neq \operatorname{first}(y)$ then $|f(x) \wedge f(y)| \leq K$.

(ii) If $a \in X$ is a bounded letter, then $f(a)=a$.

(iii) If $a \in X$ is a growing letter, then $|f(a)| \geq 2$.

In this subsection we prove Theorem 2.2 for morphisms which share properties (i)-(iii). To proceed we fix such a morphism $f: X^{*} \longrightarrow X^{*}$.

Let $a \in X$ be a growing letter and let $a_{n}$ be the first growing letter of $f^{n}(a)$ for $n \geq 0$. Then the sequence $\left(a_{n}\right)_{n \geq 0}$ is ultimately periodic. Hence we can find a positive integer $n_{0}$ such that if $a, b \in X$ are growing letters and $a_{n}=b_{n}$ for some $n \geq 0$, then $a_{n_{0}}=b_{n_{0}}$. Denote

$$
M=\max _{x \in X}\left|f^{n_{0}}(x)\right|
$$


Lemma 3.1. If $a, b \in X$ are growing letters and $z \in X^{*}$ is a bounded word with $|z| \geq M$ then $f^{n}(a) \wedge f^{n}(z b)$ is a bounded word for all $n \geq 0$.

For the proof of Lemma 3.1 we refer to [4].

Next we define a family of mappings needed for the proof of Theorem 2.2. Let $M$ be as above. Denote $N=K(M+K)$ where $K$ is as in (i). First, define the mapping $\tau_{1}: X^{*} \longrightarrow X^{*}$ as follows. If $w \in X^{*}$ contains $M+K$ consecutive bounded letters, then $\tau_{1}(w)$ is the shortest prefix of $w$ ending with $M+K$ bounded letters. Otherwise, $\tau_{1}(w)=w$. Next, the mapping $\tau_{2}: X^{*} \longrightarrow X^{*}$ is defined by

$$
\tau_{2}(w)=\operatorname{pref}_{N}(w), \quad w \in X^{*} .
$$

Then, define $\tau: X^{*} \longrightarrow X^{*}$ as follows. If $w \in X^{*}$ has a prefix of length $M$ consisting of bounded letters, $\tau(w)=\operatorname{pref}_{M}(w)$. Otherwise $\tau(w)=\tau_{2} \tau_{1}(w)$.

Next, we consider pairs of words and extend the mapping $\tau$ in a natural way by setting

$$
\tau(u, v)=(\tau(u), \tau(v)), \quad(u, v) \in X^{*} \times X^{*} .
$$

Finally, if $(u, v) \in X^{*} \times X^{*}$, define the mapping $\rho_{u, v}: \mathbf{N} \longrightarrow X^{*} \times X^{*}$ by

$$
\rho_{u, v}(n)=\tau\left(f^{n}(u) * f^{n}(v)\right), \quad n \geq 0 .
$$

Denote

$$
\Lambda_{u, v}=\left\{\rho_{u, v}(n) \mid n \in \mathbf{N}\right\} .
$$

Define the set $\mathcal{R} \subseteq X^{*} \times X^{*}$ as follows. Let $(u, v) \in X^{*} \times X^{*}$ and denote $\left(u_{n}, v_{n}\right)=f^{n}(u) * f^{n}(v)$ for $n \geq 0$. Then $(u, v) \in \mathcal{R}$ if and only if $\psi(u)=\psi(v)$ and for all $n \geq 0$ the word $u_{n}$ (resp. $v_{n}$ ) contains a growing letter and $\left|u_{n}\right| \geq N$ (resp. $\left.\left|v_{n}\right| \geq N\right)$.

Let $(u, v) \in \mathcal{R}$ and let $n \geq 0$. Write $\rho_{u, v}(n)=\left(w_{1}, w_{2}\right)$. Then the words $w_{i}$, $i=1,2$, satisfy the following conditions:

(1) $M \leq\left|w_{i}\right| \leq N$;

(2) if $\left|w_{i}\right|=M$, then $w_{i}$ is a bounded word;

(3) if $M<\left|w_{i}\right|<N$, then $w_{i}$ ends with $M+K$ bounded letters;

(4) if $\left|w_{i}\right|=N$, either $w_{i}$ does not have a bounded factor of length $M+K$ or the suffix of $w_{i}$ of length $M+K$ is the only bounded factor of length $M+K$ of $w_{i}$.

A pair $(u, v) \in X^{*} \times X^{*}$ is called special if $|u|=M$ or $|v|=M$.

Lemma 3.2. Suppose $(u, v) \in \mathcal{R}$ and $(x, y) \in \Lambda_{u, v}$. If $w_{1}, w_{2} \in X^{*}$ and $(x, y)$ is not special, then

$$
\tau(f(x) * f(y))=\tau\left(f(x) w_{1} * f(y) w_{2}\right) .
$$

For the proof of Lemma 3.2 we again refer to [4].

Lemma 3.3. Suppose $(u, v) \in \mathcal{R}$ and write $\rho_{u, v}(n)=\left(x_{n}, y_{n}\right)$ for $n \geq 0$. If none of the pairs $\left(x_{n}, y_{n}\right), n \geq 0$, is special, then

$$
\rho_{u, v}(i+t)=\tau\left(f^{t}\left(x_{i}\right) * f^{t}\left(y_{i}\right)\right)
$$


for all $i \geq 0$ and $t \geq 0$.

Proof. If $\left(w_{1}, w_{2}\right) \in X^{*} \times X^{*}$, we have $\tau\left(w_{1}, w_{2}\right)=\tau\left(\tau\left(w_{1}, w_{2}\right)\right)$. Hence

$$
\rho_{u, v}(i)=\tau\left(f^{i}(u) * f^{i}(v)\right)=\tau\left(\tau\left(f^{i}(u) * f^{i}(v)\right)\right)=\tau\left(x_{i}, y_{i}\right)
$$

for all $i \geq 0$. Consequently, (2) holds for all $i \geq 0$ if $t=0$.

Suppose then that (2) holds for a fixed pair $t \geq 0, i \geq 0$. In other words

$$
\left(x_{i+t}, y_{i+t}\right)=\tau\left(f^{t}\left(x_{i}\right) * f^{t}\left(y_{i}\right)\right) .
$$

Hence there exist words $\alpha, \beta_{1}, \beta_{2} \in X^{*}$ such that

$$
f^{t}\left(x_{i}\right)=\alpha x_{i+t} \beta_{1}, \quad f^{t}\left(y_{i}\right)=\alpha y_{i+t} \beta_{2} .
$$

Because $\tau\left(f^{i+t}(u) * f^{i+t}(v)\right)=\left(x_{i+t}, y_{i+t}\right)$, there are words $\gamma, \delta_{1}, \delta_{2} \in X^{*}$ such that

$$
f^{i+t}(u)=\gamma x_{i+t} \delta_{1}, \quad f^{i+t}(v)=\gamma y_{i+t} \delta_{2} .
$$

Now we get

$$
\begin{aligned}
\rho_{u, v}(i+t+1) & =\tau\left(f^{i+t+1}(u) * f^{i+t+1}(v)\right) \\
& =\tau\left(f\left(\gamma x_{i+t} \delta_{1}\right) * f\left(\gamma y_{i+t} \delta_{2}\right)\right) \\
& =\tau\left(f\left(x_{i+t}\right) * f\left(y_{i+t}\right)\right) \\
& =\tau\left(f(\alpha) f\left(x_{i+t}\right) f\left(\beta_{1}\right) * f(\alpha) f\left(y_{i+t}\right) f\left(\beta_{2}\right)\right) \\
& =\tau\left(f^{t+1}\left(x_{i}\right) * f^{t+1}\left(y_{i}\right)\right) .
\end{aligned}
$$

Here the third and fourth equations follow by Lemma 3.2. Consequently, (2) holds for all $i \geq 0$ and $t \geq 0$.

By Theorem 2.1 there is a positive integer $q_{1}$ such that $q_{1}$ is a period of the sequence

$$
\left(\operatorname{pref}_{p}\left(f^{n t}(a)\right)\right)_{n \geq 0}
$$

for all $p \geq 1, a \in X$ and $t<\operatorname{card}(X)^{2 N+2}$. Fix such an integer $q_{1}$. Then $q_{1}$ is also a period of

$$
\left(\operatorname{pref}_{p}\left(f^{n t}(w)\right)\right)_{n \geq 0}
$$

for all $p \geq 1, w \in X^{*}$ and $t<\operatorname{card}(X)^{2 N+2}$.

Lemma 3.4. Let $(u, v) \in \mathcal{R}$ and write $\rho_{u, v}(n)=\left(x_{n}, y_{n}\right)$ for $n \geq 0$. Suppose none of the pairs $\left(x_{n}, y_{n}\right), n \geq 0$, is special. Then $q_{1}$ is a period of (1) for any positive integer $p$.

Proof. Because the set $\Lambda_{u, v}$ has less than card $(X)^{2 N+2}$ elements, there are positive integers $i$ and $t$ such that $t<\operatorname{card}(X)^{2 N+2}$ and

$$
\rho_{u, v}(i)=\rho_{u, v}(i+t)
$$


By Lemma 3.3 we have

$$
\left(x_{i}, y_{i}\right)=\left(x_{i+t}, y_{i+t}\right)=\tau\left(f^{t}\left(x_{i}\right) * f^{t}\left(y_{i}\right)\right) .
$$

Hence there exist words $\alpha, \gamma_{1}, \gamma_{2} \in X^{*}$ such that

$$
f^{t}\left(x_{i}\right)=\alpha x_{i} \gamma_{1}, \quad f^{t}\left(y_{i}\right)=\alpha y_{i} \gamma_{2} .
$$

Let $w_{1}, w_{2} \in X^{*}$ be words such that

$$
f^{i}(u) * f^{i}(v)=\left(x_{i} w_{1}, y_{i} w_{2}\right) .
$$

Then

$$
\begin{aligned}
& f^{i+n t}(u) * f^{i+n t}(v)=f^{n t}\left(x_{i} w_{1}\right) * f^{n t}\left(y_{i} w_{2}\right) \\
& \quad=\left(x_{i} \gamma_{1} f^{t}\left(\gamma_{1}\right) \ldots f^{(n-1) t}\left(\gamma_{1}\right) f^{n t}\left(w_{1}\right), y_{i} \gamma_{2} f^{t}\left(\gamma_{2}\right) \ldots f^{(n-1) t}\left(\gamma_{2}\right) f^{n t}\left(w_{2}\right)\right)
\end{aligned}
$$

for $n \geq 1$. This implies that $q_{1}$ is a period of

$$
\left(\operatorname{pref}_{p}\left(f^{i+n t}(u) * f^{i+n t}(v)\right)\right)_{n \geq 0}
$$

for any $p \geq 1$. Because the morphism $f$ has property (i), it follows that $q_{1}$ is also a period of (1) for any $p \geq 1$.

Next, define

$$
\begin{aligned}
q_{2}= & \max \{1,|\beta| \mid \text { there are a growing letter } c \in X, \text { a positive integer } \\
& \left.s \leq \operatorname{card}(X)^{2} \text { and a word } \gamma \in X^{*} \text { such that } f^{s}(c)=\beta c \gamma\right\}
\end{aligned}
$$

and define

$$
q_{3}=q_{2} ! q_{1}
$$

Lemma 3.5. Let $(u, v) \in \mathcal{R}$ and write $\rho_{u, v}(n)=\left(x_{n}, y_{n}\right)$ for $n \geq 0$. If there is an integer $n$ such that $\left(x_{n}, y_{n}\right)$ is special, then $q_{3}$ is a period of (1) for any positive integer $p$.

Proof. Suppose that $k$ is a nonnegative integer such that $\rho_{u, v}(k)=\left(x_{k}, y_{k}\right)$ is special. Without loss of generality assume that $\left|x_{k}\right|=M$. Hence $x_{k}$ is a bounded word. Let

$$
f^{k}(u)=\alpha \beta_{1} a \gamma_{1}, \quad f^{k}(v)=\alpha \beta_{2} b \gamma_{2}
$$

where $\beta_{1}, \beta_{2} \in X^{*}$ are bounded words, $a, b \in X$ are growing letters and $\alpha, \gamma_{1}, \gamma_{2} \in$ $X^{*}$. Furthermore, $x_{k}$ is a prefix of $\beta_{1}$ and $y_{k}$ is a prefix of $\beta_{2} b \gamma_{2}$.

If $\beta_{2}$ is not empty, then

$$
f^{k+n}(u) * f^{k+n}(v)=f^{n}\left(\beta_{1} a \gamma_{1}\right) * f^{n}\left(\beta_{2} b \gamma_{2}\right)=\left(\beta_{1} f^{n}\left(a \gamma_{1}\right), \beta_{2} f^{n}\left(b \gamma_{2}\right)\right)
$$

for $n \geq 0$. Hence $q_{1}$ and $q_{3}$ are periods of (1). 
To proceed assume that $\beta_{2}$ is the empty word. Then Lemma 3.1 implies that $f^{n}\left(\beta_{1} a\right) \wedge f^{n}(b)$ is a bounded word for all $n \geq 0$. Now, there exist integers $k_{1} \geq 0$, $k_{2} \geq 1$, growing letters $c, d \in X$, bounded words $\beta_{3}, \beta_{4}, \beta_{5}, \beta_{6} \in X^{*}$ and words $\gamma_{3}, \gamma_{4}, \gamma_{5}, \gamma_{6} \in X^{*}$ such that $k_{2} \leq \operatorname{card}(X)^{2}$ and

$$
f^{k_{1}}(a)=\beta_{3} c \gamma_{3}, f^{k_{1}}(b)=\beta_{4} d \gamma_{4}, f^{k_{2}}(c)=\beta_{5} c \gamma_{5}, f^{k_{2}}(d)=\beta_{6} d \gamma_{6} .
$$

(Here we may have $c=d$.) Hence

$$
\begin{aligned}
& f^{k+k_{1}+n k_{2}}(u) * f^{k+k_{1}+n k_{2}}(v)= \\
& \quad \beta_{1} \beta_{3} \beta_{5}^{n} c \gamma_{5} f^{k_{2}}\left(\gamma_{5}\right) \ldots f^{n k_{2}-k_{2}}\left(\gamma_{5}\right) f^{n k_{2}}\left(\gamma_{3}\right) f^{k_{1}+n k_{2}}\left(\gamma_{1}\right) \\
& \quad * \beta_{4} \beta_{6}^{n} d \gamma_{6} f^{k_{2}}\left(\gamma_{6}\right) \ldots f^{n k_{2}-k_{2}}\left(\gamma_{6}\right) f^{n k_{2}}\left(\gamma_{4}\right) f^{k_{1}+n k_{2}}\left(\gamma_{2}\right)
\end{aligned}
$$

for $n \geq 1$. Furthermore, the words $\beta_{1} \beta_{3} \beta_{5}^{n} c$ and $\beta_{4} \beta_{6}^{n} d$ are not comparable. Consequently the sequence

$$
\left(\operatorname{pref}_{p}\left(f^{k+k_{1}+n k_{2}}(u) * f^{k+k_{1}+n k_{2}}(v)\right)\right)_{n \geq 0}
$$

has period $q_{3}$. In fact, if there is an integer $n$ such that $\beta_{1} \beta_{3} \beta_{5}^{n}$ and $\beta_{4} \beta_{6}^{n}$ are not comparable, (3) has period $q_{3}$. Otherwise, without loss of generality assume that $\beta_{1} \beta_{3} \beta_{5}^{n}$ is a prefix of $\beta_{4} \beta_{6}^{n}$ for all large $n$, say, if $n \geq n_{0}$. Then the sequence

$$
\left(\operatorname{pref}_{p}\left(\left(\beta_{1} \beta_{3} \beta_{5}^{n}\right)^{-1} \beta_{4} \beta_{6}^{n}\right)\right)_{n \geq n_{0}}
$$

has period $q_{2}$ ! for any $p \geq 1$. Because the sequences

$$
\left(\operatorname{pref}_{p}\left(c \gamma_{5} f^{k_{2}}\left(\gamma_{5}\right) \ldots f^{n k_{2}-k_{2}}\left(\gamma_{5}\right) f^{n k_{2}}\left(\gamma_{3}\right) f^{k_{1}+n k_{2}}\left(\gamma_{1}\right)\right)\right)_{n \geq 0}
$$

and

$$
\left(\operatorname{pref}_{p}\left(d \gamma_{6} f^{k_{2}}\left(\gamma_{6}\right) \ldots f^{n k_{2}-k_{2}}\left(\gamma_{6}\right) f^{n k_{2}}\left(\gamma_{4}\right) f^{k_{1}+n k_{2}}\left(\gamma_{2}\right)\right)\right)_{n \geq 0}
$$

have period $q_{1}$ for any $p \geq 1$, it follows that (3) has period $q_{3}$. Because $f$ has property (i) it again follows that (1) has period $q_{3}$.

We have now shown that if $f$ satisfies conditions (i)-(iii) then $q_{3}$ is a period of (1) for all $p \geq 1$ and for all $(u, v) \in \mathcal{R}$.

To proceed, let $\$$ be a new letter. Define $X_{1}=X \cup\{\$\}$ and let $f_{1}: X_{1}^{*} \longrightarrow X_{1}^{*}$ be the extension of $f$ defined by $f_{1}(\$)=\$$. Then $f_{1}$ satisfies (i)-(iii) and we know that there are positive integers $q$ and $N_{1}$ such that $q$ is a period of

$$
\left(\operatorname{pref}_{p}\left(f_{1}^{n}\left(u_{1}\right) * f_{1}^{n}\left(v_{1}\right)\right)\right)_{n \geq 0}
$$

for all $p \geq 1$ and for all $u_{1}, v_{1} \in X_{1}^{*}$ such that $\psi\left(u_{1}\right)=\psi\left(v_{1}\right)$ and each component of $f_{1}^{n}\left(u_{1}\right) * f_{1}^{n}\left(v_{1}\right)$ contains a growing letter and has length at least $N_{1}$ for all $n \geq 0$.

Let now $u, v \in X^{*}$. If there is an integer $n$ such that $f^{n}(u)$ and $f^{n}(v)$ are comparable, then (1) has period one for all $p \geq 1$. Also, if neither $u$ nor $v$ contains a growing letter, (1) has period one for all $p \geq 1$. Assume that $f^{n}(u)$ and $f^{n}(v)$ 
are incomparable for all $n \geq 0$ and assume that $u$ or $v$ contains a growing letter. Now, define

$$
u_{1}=u \$ v(u v)^{N_{1}}, \quad v_{1}=v \$ u(v u)^{N_{1}} .
$$

Then $q$ is a period of (4) for all $p \geq 1$. Consequently (1) has period $q$ for all $p \geq 1$.

This concludes the proof of Theorem 2.2 for a morphism $f$ which satisfies conditions (i)-(iii).

\subsection{Proof of Theorem 2.2 - The General CASE}

We assume that the reader is familiar with elementary morphisms (see $[1,5]$ ). In particular, recall that an elementary morphism permutes the set of bounded letters.

Let $f: X^{*} \longrightarrow X^{*}$ be a morphism. Then there exist a positive integer $j_{1}$, an alphabet $Y$ and morphisms $g_{1}: X^{*} \longrightarrow Y^{*}, g_{2}: Y^{*} \longrightarrow X^{*}$ such that

$$
f^{j_{1}}=g_{2} g_{1}
$$

and the morphisms $g_{2}$ and $g_{1} g_{2}$ are elementary (see Th. III 2.2 in [5]). Consider the morphism $g_{1} g_{2}: Y^{*} \longrightarrow Y^{*}$. There exists a positive integer $j_{2}$ such that

$$
\left(g_{1} g_{2}\right)^{j_{2}}(y)=y
$$

if $y \in Y$ is a bounded letter with respect to $g_{1} g_{2}$ and

$$
\left|\left(g_{1} g_{2}\right)^{j_{2}}(y)\right| \geq 2
$$

if $y \in Y$ is a growing letter with respect to $g_{1} g_{2}$. Define $g=\left(g_{1} g_{2}\right)^{j_{2}}$ and $j=j_{1} j_{2}$. Then

for $n \geq 0$.

$$
f^{n j+j_{1}}=g_{2} g^{n} g_{1}
$$

Because $g$ satisfies conditions (i)-(iii) of the previous subsection, there is a positive integer $q$ such that $q$ is a period of

$$
\left(\operatorname{pref}_{p}\left(g^{n} g_{1} f^{i}(u) * g^{n} g_{1} f^{i}(v)\right)\right)_{n \geq 0}
$$

for all $p \geq 1, i=0,1, \ldots, j-1$ and $u, v \in X^{*}$. Because $g_{2}$ is elementary, $q$ is also a period of

$$
\left(\operatorname{pref}_{p}\left(g_{2} g^{n} g_{1} f^{i}(u) * g_{2} g^{n} g_{1} f^{i}(v)\right)\right)_{n \geq 0}
$$

for all $p \geq 1, i=0,1, \ldots, j-1$ and $u, v \in X^{*}$. In other words, $q$ is also a period of

$$
\left(\operatorname{pref}_{p}\left(f^{n j+j_{1}+i}(u) * f^{n j+j_{1}+i}(v)\right)\right)_{n \geq 0}
$$

for all $p \geq 1, i=0,1, \ldots, j-1$ and $u, v \in X^{*}$. Hence $j q$ is a period of (1) for all $p \geq 1$ and $u, v \in X^{*}$.

This concludes the proof of Theorem 2.2 in the general case. 


\section{REFERENCES}

[1] A. Ehrenfeucht and G. Rozenberg, Elementary homomorphisms and a solution of the D0L sequence equivalence problem. Theoret. Comput. Sci. 7 (1978) 169-183.

[2] A. Ehrenfeucht, K.P. Lee and G. Rozenberg, Subword complexities of various classes of deterministic developmental languages without interactions. Theoret. Comput. Sci. 1 (1975) $59-75$.

[3] G.T. Herman and G. Rozenberg, Developmental Systems and Languages. North-Holland, Amsterdam (1975).

[4] J. Honkala, The equivalence problem for DF0L languages and power series. J. Comput. Syst. Sci. 65 (2002) 377-392.

[5] G. Rozenberg and A. Salomaa, The Mathematical Theory of L Systems. Academic Press, New York (1980).

[6] G. Rozenberg and A. Salomaa (Eds.), Handbook of Formal Languages. Vol. 1-3, Springer, Berlin (1997).

[7] A. Salomaa, Jewels of Formal Language Theory. Computer Science Press, Rockville, Md. (1981).

Communicated by C. Choffrut.

Received August 30, 2005. Accepted September 13, 2006. 